Institute for Computational Mathematics

Hong Kong Baptist University

ICM Research Report

08-07 


\title{
Gains from Diversification on convex combinations : A Majorization and Stochastic Dominance Approach *
}

\author{
Martin Egozcue \\ Department of Economics FCS, Universidad de la Republica del Uruguay \\ Wing-Keung Wong \\ Department of Economics, Hong Kong Baptist University
}

September 18, 2008

Corresponding author: Wing-Keung Wong, Department of Economics, Hong Kong Baptist University WLB, Shaw Campus, Kowloon Tong, Hong Kong; Telephone: (852)-34117542, Fax: (852)-3411-5580, Email: awong@hkbu.edu.hk

${ }^{*}$ The authors are grateful to Professor Robert Graham Dyson and anonymous referees for their substantive comments that have significantly improved this manuscript. The authors would like to thank Professors Esfandiar Maasoumi and Franco Pellerey for their valuable comments that have significantly improved this manuscript. Our deepest thanks are also given to Professor Juan Dubra for his helpful comments and assistance in the paper. The second author would like to thank Professors Robert B. Miller and Howard E. Thompson for their continuous guidance and encouragement. This research is partially supported by grants from Universidad de la Republica del Uruguay and Hong Kong Baptist University. 


\title{
Gains from Diversification on convex combinations: A Majorization and Stochastic Dominance Approach
}

\begin{abstract}
By incorporating both majorization theory and stochastic dominance theory, this paper presents a general theory and a unifying framework for determining the diversification preferences of risk-averse investors and conditions under which they would unanimously judge a particular asset to be superior. In particular, we develop a theory for comparing the preferences of different convex combinations of assets that characterize a portfolio to give higher expected utility by second-order stochastic dominance. Our findings also provide additional methodology for determining the second-order stochastic dominance efficient set.
\end{abstract}

Keywords: majorization; stochastic dominance; portfolio selection; expected utility; diversification

JEL Classification: D81, G11 


\section{Introduction}

The pioneer work of Markowitz (1952) and Tobin (1958) on the mean-variance (MV) portfolio selection is a milestone in modern finance theory for optimal portfolio construction, asset allocation, and investment diversification. ${ }^{1}$ In the procedure, investors respond to the uncertainty of an investment by selecting a portfolio that maximizes anticipated profit subject to achieving a specified level of calculated risk or, equivalently, minimizes variance subject to obtaining a predetermined level of expected gain. However, the disadvantage of using the MV criterion ${ }^{2}$ is that it is derived by assuming the Von Neumann-Morgenstern (1944) quadratic utility function and returns being examined are required to be normally distributed (Feldstein, 1969; Hanoch and Levy, 1969).

To circumvent the limitations of the MV criterion, academics recommend adopting the stochastic dominance (SD) approach, which can be used in constructing a general framework for the analysis of choice and problems of diversification for risk-averse investors under uncertainty without any restriction on the distribution of the assets being analyzed and without imposing the quadratic utility function assumption on investors. Academics have regarded the SD approach as one of the most useful tools for ranking uncertain investment prospects or portfolios because their rankings have been theoretically justified to be equal to the rankings of the corresponding expected utilities. Hanoch and Levy (1969) link stochastic dominance to a class of utility functions for non-satiable and riskaverse investors. Hadar and Russell (1971) develop the analysis using the concept of stochastic dominance and its applicability to choices under conditions of uncertainty,

\footnotetext{
${ }^{1}$ To enhance the mean-variance portfolio selection, recently Leung and Wong (2008) apply the technique of the repeated measures design to develop a multivariate Sharpe ratio statistic to test the hypothesis of the equality of multiple Sharpe ratios whereas Bai, et al (2009) develop new bootstrap-corrected estimations for the optimal return and its asset allocation and prove that these bootstrap-corrected estimates are proportionally consistent with their theoretic counterparts.

${ }^{2}$ This rule provides an excellent approximation to any risk averse utility function under some restrictions on the range of return, see Levy and Markovitz (1979) for more information.
} 
whereas Tesfatsion (1976) further extends their results for diversification using a stochastic dominance approach to maximizing investors' expected utilities. Readers may refer to Ortobelli Lozza (2001) and Post (2008) for an exhaustive overview of other useful results along these lines.

By combining majorization theory with stochastic dominance theory, we extend the theory by developing some new results for choice in portfolio diversification. To specify, we establish some new theorems to determine the preferences of risk-averse investors among different diversified portfolios and show the conditions under which all risk-averse investors would prefer more diversified portfolios to less diversified ones. Our findings are important because they permit investors to specialize the rankings, by second-order stochastic dominance, from among a wide range of convex combinations of assets, and especially because they have implications concerning the weights of allocations. Our findings enable investors to make choices about allocations from their capital that result in higher expected utilities. This was one of the topics that Levy (2006) suggested for future research.

In addition, our findings could also be used in determining the second-order stochastic dominance efficient set. Traditionally, there are two decision stages in determining the efficient set; see Bawa et al. (1985). In the first stage, the initial screening of prospects or investments is accomplished by partitioning the feasible set into the efficient and inefficient sets using a stochastic dominance relation. ${ }^{3}$ At the second stage, Fishburn's (1974) concept of convex stochastic dominance (CSD) is used to eliminate elements that are not optimal in the sense of CSD. Alternatives that are dominated by convex combinations of other portfolios will be eliminated from the efficient set as they are classified to be inefficient. In this context, our findings allow investors to rank convex combinations of assets by majorization order, which, in turn, implies the rankings of their preferences of

\footnotetext{
${ }^{3}$ Readers may refer to Broll, et al. (2006), Wong (2006, 2007) and Wong and Chan (2008) and the references there for more information.
} 
second-order stochastic dominance. Thus, our findings assist investors in determining the second-order stochastic dominance efficient set.

Our paper is organized as follows. We begin by introducing definitions and notations and stating some basic properties for the majorization theory and stochastic dominance theory. Section 3 presents our findings on the preferences for risk-averse investors in their choice of diversified portfolios, and Section 4 offers some conclusions.

\section{Definitions and Notations}

In this section, we will first introduce some notations and well-known properties in stochastic dominance theory and majorization theory that we will use in this paper. Considering an economic agent with unitary initial capital, in this paper we study the single period portfolio selection for risk-averse investors to allocate their wealth to the $n(n>1)$ risks without short selling in order to maximize their expected utilities from the resulting final wealth. Let random variable $X$ be an (excess) return of an asset or prospect. If there are $n$ assets $\vec{X}_{n}=\left(X_{1}, \cdots, X_{n}\right)^{\prime},{ }^{4}$ a portfolio of $\vec{X}_{n}$ without short selling is defined by a convex combination, ${\overrightarrow{\lambda_{n}}}^{\prime} \vec{X}_{n}$, of the $n$ assets $\vec{X}_{n}$ for any $\overrightarrow{\lambda_{n}} \in S_{n}^{0}$ where

$$
S_{n}^{0}=\left\{\left(s_{1}, s_{2}, \cdots, s_{n}\right)^{\prime} \in \mathbb{R}^{n}: 0 \leq s_{i} \leq 1 \text { for any } i, \sum_{i=1}^{n} s_{i}=1\right\}
$$

in which $\mathbb{R}$ is the set of real numbers. The $i^{\text {th }}$ element of $\overrightarrow{\lambda_{n}}$ is the weight of the portfolio allocation on the $i^{\text {th }}$ asset of return $X_{i}$. A portfolio will be equivalent to return on asset $i$ if $s_{i}=1$ and $s_{j}=0$ for all $j \neq i$. It is diversified if there exists $i$ such that $0<s_{i}<1$, and is completely diversified if $0<s_{i}<1$ for all $i=1,2, \cdots, n$. As we study the properties

\footnotetext{
${ }^{4}$ One could easily extend the theory developed in this paper to $\vec{X}_{\infty}$. We skip the discussion of theory for $\vec{X}_{\infty}$ and restrict $n$ to be a finite integer in this paper.
} 
of majorization in this context, without loss of generality, we further assume $S_{n}$ satisfy:

$$
S_{n}=\left\{\left(s_{1}, s_{2}, \cdots, s_{n}\right)^{\prime} \in \mathbb{R}^{n}: 1 \geq s_{1} \geq s_{2} \geq \cdots \geq s_{n} \geq 0, \sum_{i=1}^{n} s_{i}=1\right\}
$$

We note that the condition of $\sum_{i=1}^{n} s_{i}=1$ is not necessary. It could be any positive number in most of the findings in this paper. For convenience, we set $\sum_{i=1}^{n} s_{i}=1$ so that the sum of all relative weights is equal to one. In this paper, we will mainly study the properties of majorization by considering $\overrightarrow{\lambda_{n}} \in S_{n}$ instead of $S_{n}^{0}$.

Suppose that an investor has utility function $u$, and his/her expected utility for the portfolio $\vec{\lambda}_{n}{ }^{\prime} \vec{X}_{n}$ is $E\left[u\left({\overrightarrow{\lambda_{n}}}^{\prime} \vec{X}_{n}\right)\right]$. In this context, we study only the behavior of nonsatiable and risk-averse investors whose utility functions belong to the following classes (see, for example, Ingersoll, 1987):

Definition $1^{5} \quad U_{2}$ is the set of the utility functions, u, defined in $\mathbb{R}$ such that:

$$
U_{2}=\left\{u:(-1)^{i+1} u^{(i)} \geq 0, i=1,2\right\}
$$

where $u^{(i)}$ is the $i^{\text {th }}$ derivative of the utility function $u$, and the extended set of utility functions is:

$$
U_{2}^{E}=\{u: u \text { is increasing and concave }\}
$$

We note that in the above definition, "increasing" means "non-decreasing." It is known (e.g., see Theorem $11 \mathrm{C}$ in Roberts and Varberg 1973) that $u$ in $U_{2}^{E}$ is differentiable almost everywhere and its derivative is continuous almost everywhere. We note that the theory can be easily extended to satisfy utilities defined in Definition 1 to be non-differentiable. ${ }^{6}$

\footnotetext{
${ }^{5}$ We note that if $u \in U_{2}, u$ is Fréchet differentiable; see, for example, Machina (1982) for more information

${ }^{6}$ Readers may refer to Wong and $\mathrm{Ma}(2008)$ and the references there for more information. In this paper, we will skip the discussion of non-differentiable utilities.
} 
There are many ways to order the elements in $S_{n}$. A popular one is to order them by majorization; see, for example, Hardy, et al. (1934) and Marshall and Olkin (1979), as stated in the following:

Definition 2 Let $\vec{\alpha}_{n}, \vec{\beta}_{n} \in S_{n}$ in which $S_{n}$ is defined in (2). $\vec{\beta}_{n}$ is said to majorize $\vec{\alpha}_{n}$, denoted by $\vec{\beta}_{n} \succeq_{M} \vec{\alpha}_{n}$, if $\sum_{i=1}^{k} \beta_{i} \geq \sum_{i=1}^{k} \alpha_{i}$, for all $k=1,2, \cdots, n$.

Majorization is a partial order among vectors of real numbers. We illustrate it in the following example:

Example $1 \quad\left(\frac{3}{5}, \frac{1}{5}, \frac{1}{5}\right)^{\prime} \succeq_{M}\left(\frac{2}{5}, \frac{2}{5}, \frac{1}{5}\right)^{\prime}$ because $\frac{3}{5}>\frac{2}{5}$ and $\frac{3}{5}+\frac{1}{5} \geq \frac{2}{5}+\frac{2}{5}$.

Vectors that can be ordered by majorization have some interesting properties. One of them is Dalton Pigou transfer, as described in the following definition:

Definition $3^{7}$ For any $\vec{\alpha}_{n}, \vec{\beta}_{n} \in S_{n}, \vec{\alpha}_{n}$ is said to be obtained from $\vec{\beta}_{n}$ by applying a single Dalton (Pigou) transfer, denoted by $\vec{\beta}_{n} \stackrel{d}{\rightarrow} \vec{\alpha}_{n}$, if there exist $h$ and $k(1 \leq h<k \leq n)$ such that $\alpha_{i}=\beta_{i}$ for any $i \neq h, k ; \alpha_{h}=\beta_{h}-\epsilon$; and $\alpha_{k}=\beta_{k}+\epsilon$ with $\epsilon>0$.

For instance, consider the above example that $\vec{\alpha}_{3}=\left(\frac{2}{5}, \frac{2}{5}, \frac{1}{5}\right)^{\prime}$ and $\vec{\beta}_{3}=\left(\frac{3}{5}, \frac{1}{5}, \frac{1}{5}\right)^{\prime}$, As $\alpha_{1}=\beta_{1}-\frac{1}{5}, \alpha_{2}=\beta_{2}+\frac{1}{5}$, and $\alpha_{3}=\beta_{3}=\frac{1}{5}$, from Definition 3 , we said that $\vec{\alpha}_{3}$ can be obtained from $\vec{\beta}_{3}$ by applying a single Dalton transfer by setting $\alpha_{1}=\beta_{1}-\frac{1}{5}$ and $\alpha_{2}=\beta_{2}+\frac{1}{5}$. Thus, we write $\vec{\beta}_{3} \stackrel{d}{\rightarrow} \vec{\alpha}_{3}$.

In this example, we also notice that $\vec{\beta}_{3}$ majorizes $\vec{\alpha}_{3}$. One may wonder whether there is any relationship between majorization and Dalton transfer. To answer this question, we have the following theorem:

Theorem 1 Let $\vec{\alpha}_{n}, \vec{\beta}_{n} \in S_{n}, \vec{\beta}_{n} \succeq_{M} \vec{\alpha}_{n}$ if and only if $\vec{\alpha}_{n}$ can be obtained from $\vec{\beta}_{n}$ by applying a finite number of Dalton transfers, denoted by $\vec{\beta}_{n} \stackrel{D}{\rightarrow} \vec{\alpha}_{n}$.

\footnotetext{
${ }^{7}$ Some academic suggest the reverse direction for the definition of Dalton Pigou transfer. In this paper, we follow Ok and Kranich (1998) for the definition.
} 
This theorem states that if $\vec{\beta}_{n}$ majorizes $\vec{\alpha}_{n}$, then $\vec{\alpha}_{n}$ can be obtained from $\vec{\beta}_{n}$ by applying a finite number of single Dalton transfers, and vice versa. We illustrate the procedure in the following example:

Example 2 Consider $\left(\frac{1}{3}, \frac{1}{3}, \frac{1}{3}\right)^{\prime}$ and $\left(\frac{4}{5}, \frac{1}{5}, 0\right)^{\prime}$. As $\left(\frac{1}{3}, \frac{1}{3}, \frac{1}{3}\right)^{\prime}$ is majorized by $\left(\frac{4}{5}, \frac{1}{5}, 0\right)^{\prime}$, from Theorem 1, we know that $\left(\frac{1}{3}, \frac{1}{3}, \frac{1}{3}\right)^{\prime}$ can be obtained by applying a finite number of single Dalton transfers on $\left(\frac{4}{5}, \frac{1}{5}, 0\right)^{\prime}$. This could be done, for example, by setting $\left(\frac{4}{5}, \frac{1}{5}, 0\right)^{\prime} \stackrel{d}{\rightarrow}\left(\frac{2}{3}, \frac{1}{3}, 0\right)^{\prime} \stackrel{d}{\rightarrow}\left(\frac{1}{3}, \frac{1}{3}, \frac{1}{3}\right)^{\prime}$. That is, by simply first transferring $\frac{2}{15}$ from the first entry to the second entry of $\left(\frac{4}{5}, \frac{1}{5}, 0\right)^{\prime}$ and then transferring $\frac{1}{3}$ from its first entry to its third entry to obtain $\left(\frac{1}{3}, \frac{1}{3}, \frac{1}{3}\right)^{\prime}$. Thus, we write $\left(\frac{4}{5}, \frac{1}{5}, 0\right)^{\prime} \stackrel{D}{\rightarrow}\left(\frac{1}{3}, \frac{1}{3}, \frac{1}{3}\right)^{\prime}$.

In this paper, we link Dalton transfer and majorization order to stochastic dominance. The theory of stochastic dominance is important in decision making, since the rankings of assets or portfolio preferences have been proved to be equivalent to the rankings of their corresponding expected utilities. Before we discuss stochastic dominance, we define some notations as follows. Let $\overline{\mathbb{R}}$ be the set of extended real numbers. Suppose that $\Omega=[a, b]$ is a subset of $\overline{\mathbb{R}}$ in which $a$ and $b$ can be finite or infinite. Let $\mathbb{B}$ be the Borel $\sigma$-field of $\Omega$ and $\mu$ be a measure on $(\Omega, \mathbb{B})$. The function $F$ of the measure $\mu$ is defined as:

$$
F(x)=\mu(a, x] \text { for all } x \in \Omega \text {. }
$$

The function $F$ is called a (probability) distribution function and $\mu$ is called a probability measure if $\mu(\Omega)=1$. By the basic probability theory, for any random variable $X$ and for probability measure $P$, there exists a unique induced probability measure $\mu$ on $(\Omega, \mathbb{B})$ and the probability distribution function $F$ such that $F$ satisfies (3) and

$$
\mu(B)=P\left(X^{-1}(B)\right)=P(X \in B) \quad \text { for any } \quad B \in \mathbb{B} .
$$

An integral written in the form of $\int_{A} f(t) d \mu(t)$ or $\int_{A} f(t) d F(t)$ is a Lebesgue-Stieltjes integral for any integrable function $f(t)$. If the integral has the same value for any set $A$ 
that is equal to $(c, d],[c, d)$ or $[c, d]$, then we use the notation $\int_{c}^{d} f(t) d \mu(t)$ instead. In addition, if $\mu$ is a Borel measure with $\mu(c, d]=d-c$ for any $c<d$, then we write the integral as $\int_{c}^{d} f(t) d t$. The Lebesgue-Stieltjes integral $\int_{c}^{d} f(t) d t$ is equal to the Riemann integral if $f$ is bounded and continuous almost everywhere on $[c, d]$; see Theorem 1.7.1 in Ash (1972).

We consider random variables, denoted by $X, Y, \cdots$ defined on $\Omega$. The probability distribution functions of $X$ and $Y$ are $F$ and $G$, respectively. Throughout this paper, all functions are assumed to be measureable and all integrals and expectations are implicitly assumed to exist and to be finite. We next define the second-order stochastic dominance that will be useful for risk-averse investors in making their decision as follows:

Definition 4 Given two random variables $X$ and $Y$ with $F$ and $G$ as their respective probability distribution functions defined on $[a, b], X$ dominates $Y$ and $F$ dominates $G$ in the sense of $S S D$, denoted by $X \succeq_{2} Y$ or $F \succeq_{2} G$, if and only if $\int_{a}^{x} F(y) d y \leq \int_{a}^{x} G(y) d y$ for each $x$ in $[a, b]$, where SSD stands for second-order stochastic dominance.

An individual chooses between $F$ and $G$ in accordance with a consistent set of preferences satisfying the Von Neumann-Morgenstern (1944) consistency properties. Accordingly, $F$ is preferred to $G$, or equivalently, $X$ is preferred to $Y$ for all utility functions $u$ if

$$
\Delta E u \equiv E[u(X)]-E[u(Y)] \geq 0
$$

where $E[u(X)] \equiv \int_{a}^{b} u d F$ and $E[u(Y)] \equiv \int_{a}^{b} u d G$.

\section{The Theory}

In this section, we will develop the theory of diversification for risk-averse investors to make comparisons among different portfolios by incorporating both majorization theory and stochastic dominance theory. 
We first discuss the stochastic dominance theory for random variables, and nonnegative combinations, or equivalently convex combinations, of random variables. Random variables $X, Y, \cdots$ can be regarded as returns on individual prospects and convex combinations of random variables can be regarded as the returns on the portfolios for different prospects. Hence, stochastic dominance for the random variables can be applied to examine preferences of different prospects and the preferences of different portfolios. The theory of stochastic dominance is important because it is equivalent to the theory of utility maximization as stated in the following theorem:

Theorem 2 Let $X$ and $Y$ be random variables with probability distribution functions $F$ and $G$, respectively. Suppose $u$ is a utility function. Then,

$$
X \succeq_{2} Y \text { or, equivalently, } F \succeq_{2} G \text { if and only if } E[u(X)] \geq E[u(Y)]
$$

for any $u \in U_{2}^{E}$.

We note that Hanoch and Levy (1969) and Hadar and Russell (1969) first prove this theorem. Readers may refer to their papers for the proof of the theorem.

Hadar and Russel (1971) first investigate the diversification problem for the independent and identically distributed case in a bivariate setting. They verify that

$$
E\left[u\left({\overrightarrow{\alpha_{2}}}^{\prime} \vec{X}_{2}\right)\right] \geq E\left[u\left({\overrightarrow{\beta_{2}}}^{\prime} \vec{X}_{2}\right)\right]
$$

whenever $\left|\alpha_{1}-\alpha_{2}\right| \leq\left|\beta_{1}-\beta_{2}\right|$ where $\vec{\alpha}_{2}=\left(\alpha_{1}, \alpha_{2}\right)^{\prime}$ and $\vec{\beta}_{2}=\left(\beta_{1}, \beta_{2}\right)^{\prime} \in S_{2}, u \in U_{2}$, and $\vec{X}_{2}=\left(X_{1}, X_{2}\right)^{\prime}$ in which $X_{1}$ and $X_{2}$ are non-negative independent and identically distributed random variables.

We note that for any pair of random variables $X$ and $Y$, the statements $X \succeq_{2} Y$ and $F \succeq_{2} G$ are equivalent. But for $n>1$, the statements $\vec{\alpha}_{n}^{\prime} \vec{X}_{n} \succeq_{2} \vec{\beta}_{n}^{\prime} \vec{Y}_{n}$ and $\vec{\alpha}_{n}^{\prime} \vec{F}_{n} \succeq_{2}$ 
$\vec{\beta}_{n}^{\prime} \vec{G}_{n}^{8}$ are different because the distribution functions of $\vec{\alpha}_{n}^{\prime} \vec{X}_{n}$ and $\vec{\beta}_{n}^{\prime} \vec{Y}_{n}$ are different from $\vec{\alpha}_{n}^{\prime} \vec{F}_{n}$ and $\vec{\beta}_{n}^{\prime} \vec{G}_{n}$, respectively. Thus, we cannot apply the convex stochastic dominance theorems obtained in Fishburn (1974), Dekel (1989), and Wong and Li (1999) to the convex combinations of random variables. To investigate the properties of the convex combinations of random variables, Hadar and Russell (1971) and Tesfatsion (1976) first study the invariance property of the stochastic dominance for the convex combinations of random variables in a bivariate setting, whereas Li and Wong (1999) further extend their work by comparing two sets of independent variables in a multivariate setting as shown in the following theorem:

Theorem 3 For $n>1$, let $\vec{X}_{n}=\left(X_{1}, \cdots, X_{n}\right)^{\prime}$ and $\vec{Y}_{n}=\left(Y_{1}, \cdots, Y_{n}\right)^{\prime}$ where $\left\{X_{1}, \cdots, X_{n}\right\}$ and $\left\{Y_{1}, \cdots, Y_{n}\right\}$ be two sets of independent and identically distributed random variables. Then, $X_{i} \succeq_{2} Y_{i}$ for any $i=1,2, \cdots, n$ if and only if $\vec{\alpha}_{n}^{\prime} \vec{X}_{n} \succeq_{2} \vec{\alpha}_{n}^{\prime} \vec{Y}_{n}$ for any $\vec{\alpha}_{n} \in S_{n}^{0}$.

One may refer to Li and Wong (1999) for the proof. We provide the following example to illustrate Theorem 3:

Example 3 Consider $Y_{i}$ to be independently distributed with zero mean and unit variance and $X_{i}=a+b Y_{i}$ with $a \geq 0$ and $0<b \leq 1$ for any $i=1, \cdots, n$. Applying Theorem 8 in Li and Wong (1999), one could show that $X_{i} \succeq_{2} Y_{i}$ for any $i=1,2, \cdots, n$. Thereafter, applying Theorem 3, one could obtain $\vec{\alpha}_{n}^{\prime} \vec{X}_{n} \succeq_{2} \vec{\alpha}_{n}^{\prime} \vec{Y}_{n}$ for any $\vec{\alpha}_{n} \in S_{n}^{0}$.

Let $\left(\frac{\overrightarrow{1}}{n}\right)=\left(\frac{1}{n}, \cdots, \frac{1}{n}\right)^{\prime}$. Hanoch and Levy (1969), Hadar and Russell (1971), and Tesfatsion (1976) verify in the bivariate case that, for any $\left(\frac{\overrightarrow{1}}{2}\right)$ and $\vec{\alpha}_{2} \in S_{2}^{0}$, if $X_{1}$ and $X_{2}$ are independent and identically distributed, then

$$
\left(\frac{\overrightarrow{1}}{2}\right)^{\prime} \vec{X}_{2} \succeq_{2} \vec{\alpha}_{2}^{\prime} \vec{X}_{2} \succeq_{2} X_{i} \text { for } i=1,2
$$

${ }^{8} \vec{X}_{n}=\left(X_{1}, \cdots, X_{n}\right)^{\prime}, \vec{Y}_{n}=\left(Y_{1}, \cdots, Y_{n}\right)^{\prime}, \vec{F}_{n}=\left(F_{1}, \cdots, F_{n}\right)^{\prime}$ and $\vec{G}_{n}=\left(G_{1}, \cdots, G_{n}\right)^{\prime}$ where $F_{i}$ and $G_{i}$ are the distribution functions of $X_{i}$ and $Y_{i}$, respectively. 
$\mathrm{Li}$ and Wong (1999) generalize the results further to the case of random variables in a multivariate setting as shown in the following theorem:

Theorem 4 For $n>1$, let $\vec{X}_{n}=\left(X_{1}, \cdots, X_{n}\right)^{\prime}$ where $X_{1}, \cdots, X_{n}$ are independent and identically distributed, then, for any $i=1,2, \cdots, n$ and for any $\left(\frac{\overrightarrow{1}}{n}\right), \vec{\alpha}_{n} \in S_{n}^{0}$,

$$
\left(\frac{\overrightarrow{1}}{n}\right)^{\prime} \vec{X}_{n} \succeq_{2} \vec{\alpha}_{n}^{\prime} \vec{X}_{n} \succeq_{2} X_{i}
$$

Readers may refer to $\mathrm{Li}$ and Wong (1999) for the proof of Theorem 4. We note that Samuelson (1967) has proved that $\left(\frac{1}{n}\right)^{\prime} \vec{X}_{n}$ attains maximum among $\vec{\alpha}_{n}^{\prime} \vec{X}_{n}$ for any iid non-negative $X_{i}$. This theorem verifies the optimality of diversification that the maximal expected utility will be achieved in an equally weighted portfolio of independent and identically distributed assets. The manifestation of the generality of the theorem is that it places very weak restrictions on the weights. This theorem implies that for independent and identically distributed assets, risk-averse investors will prefer the equally weighted portfolio to any convex combination portfolio, which, in turn, is preferred to any individual asset.

Nonetheless, Theorem 4 does not permit investors to compare the preferences of other different convex combinations of random variables. So far, the comparison of the preferences of different convex combinations of random variables has not been well studied in the literature. To bridge the gap in the literature, in this paper we will develop a theory to compare the preferences of different convex combinations of random variables. We first examine the situation in which the underlying assets are independent and identically distributed. To make our contribution clear, we first state the situation in a bivariate setting as shown in the following theorem:

Theorem 5 Let $\vec{\alpha}_{2}, \vec{\beta}_{2} \in S_{2}$ and $\vec{X}_{2}=\left(X_{1}, X_{2}\right)^{\prime}$ where $X_{1}$ and $X_{2}$ are independent and identically distributed. Then,

$$
\vec{\beta}_{2} \succeq_{M} \vec{\alpha}_{2} \text { if and only if } \vec{\alpha}_{2}^{\prime} \vec{X}_{2} \succeq_{2} \vec{\beta}_{2}^{\prime} \vec{X}_{2} \text {. }
$$


This theorem provides a methodology for investors to make comparisons among a wide range of portfolios so that they make better choices in their investment decisions, especially the implications concerning the weights of allocations.

It is interesting to note from Theorem 1 that, if $\vec{\alpha}_{2}$ is majorized by $\vec{\beta}_{2}, \vec{\alpha}_{2}$ can be obtained from vector $\vec{\beta}_{2}$ by applying Dalton transfer(s) and vice versa. Thus, we could incorporate Theorem 1 into Theorem 5 to obtain the following corollary:

Corollary 6 Let $\vec{\alpha}_{2}, \vec{\beta}_{2} \in S_{2}$ and $\vec{X}_{2}=\left(X_{1}, X_{2}\right)^{\prime}$ where $X_{1}$ and $X_{2}$ are independent and identically distributed. Then,

$$
\vec{\beta}_{2} \stackrel{D}{\rightarrow} \vec{\alpha}_{2} \quad \text { if and only if } \quad \vec{\alpha}_{2}^{\prime} \vec{X}_{2} \succeq_{2} \vec{\beta}_{2}^{\prime} \vec{X}_{2}
$$

Theorem 5 and Corollary 6 extend the results developed by Hadar and Russell (1971), Tesfatsion (1976), and Li and Wong (1999). In this context, we further generalize the above results to a multivariate setting as shown in the following theorem:

Theorem 7 For $n>1$, let $\vec{\alpha}_{n}, \vec{\beta}_{n} \in S_{n}{ }^{9}$ and $\vec{X}_{n}=\left(X_{1}, \cdots, X_{n}\right)^{\prime}$ where $X_{1}, \cdots, X_{n}$ are independent and identically distributed. If $\vec{\beta}_{n} \succeq_{M} \vec{\alpha}_{n}$, then $\vec{\alpha}_{n}^{\prime} \vec{X}_{n} \succeq_{2} \vec{\beta}_{n}^{\prime} \vec{X}_{n}$.

The relationship between stochastic dominance and majorization order characterized by this theorem allows us to rank different convex combinations of two sets of independent and identically distributed assets in a multivariate setting. It conveys two messages to investors: First, any risk-averse investor will always prefer portfolios with majorized vectors of allocations to ones with majorizing vectors of allocations. Second, if $\vec{\alpha}_{n}$ and $\vec{\beta}_{n}$ cannot be ranked by majorization, the portfolios $\vec{\alpha}_{n}^{\prime} \vec{X}_{n}$ and $\vec{\beta}_{n}^{\prime} \vec{X}_{n}$ could be SSD incomparable. ${ }^{10}$ Further investigation of their SSD determination is required.

\footnotetext{
${ }^{9}$ We keep the condition $\sum_{i=1}^{n} s_{i}=1$ in $S_{n}$ for convenience. One could exclude this condition and relax it to be $\overrightarrow{1}_{n}^{\prime} \vec{\alpha}_{n}=\overrightarrow{1}_{n}^{\prime} \vec{\beta}_{n}$.

${ }^{10} X$ and $Y$ are SSD comparable if either $X \succeq_{n} Y$ or $Y \succeq_{n} X$ holds, and $X$ and $Y$ are not SSD comparable, or are SSD incomparable, if both $X \nsucceq_{n} Y$ and $Y \nsucceq_{n} X$ hold, where $X \nsucceq_{n} Y$ means $X \succeq_{n} Y$ does not hold.
} 
In addition, incorporating Theorem 1 into Theorem 7, we obtain the following corollary:

Corollary 8 For $n>1$, let $\vec{\alpha}_{n}, \vec{\beta}_{n} \in S_{n}$ and $\vec{X}_{n}=\left(X_{1}, \cdots, X_{n}\right)$ where $X_{1}, \cdots, X_{n}$ are independent and identically distributed. If $\vec{\beta}_{n} \stackrel{D}{\rightarrow} \vec{\alpha}_{n}$, then $\vec{\alpha}_{n}^{\prime} \vec{X}_{n} \succeq_{2} \vec{\beta}_{n}^{\prime} \vec{X}_{n}$.

We note that the necessary condition of Theorem 5 and Corollary 6 can only hold in a bivariate setting but not in a multivariate setting. We illustrate by the following example that the converse of Theorem 7 does not hold:

Example 4 Consider $U=\frac{1}{2} X_{1}+\frac{1}{2} X_{2}$ and $V=\frac{3}{4} X_{1}+\frac{1}{8} X_{2}+\frac{1}{8} X_{3}$ where $X_{i}(i=1,2,3)$ is independent and identically distributed as $N(0,1)$. One can easily show that $U \succeq_{2} V$ as $E(U)=E(V)$ and $\operatorname{Var}(U)<\operatorname{Var}(V)$. Nonetheless, $\left(\begin{array}{llll}\frac{1}{2} & \frac{1}{2} & 0\end{array}\right)$ and $\left(\frac{3}{4} \frac{1}{8} \frac{1}{8}\right)$ cannot be ordered by majorization.

Similarly, one could easily construct an example to show that the necessary condition of Corollary 8 does not hold in a multivariate setting. Theorem 7 and Corollary 8 further extend the results developed by Hadar and Russell (1971), Tesfatsion (1976), and Li and Wong (1999) by providing an additional methodology for investors to make comparisons among a wide range of different convex combinations of assets in a multivariate setting to check their SSD preferences. These results are very useful. They permit investors to rank different convex combinations of independent and identically distributed random variables by second-order stochastic dominance and by their corresponding vectors of allocations. Since the portfolios with majorizing vectors will be second-order stochastically dominated by the ones with majorized vectors, those with majorizing vectors should be eliminated from the SSD efficient set. Thus, our findings could be used in determining the secondorder stochastic dominance efficient set. We illustrate how to compare different portfolios in the following example:

Example 5 Suppose that one would like to rank the following assets by second-order stochastic dominance: $\vec{\alpha}_{3}^{\prime} \vec{X}_{3}=\frac{2}{5} X_{1}+\frac{2}{5} X_{2}+\frac{1}{5} X_{3}$ and $\vec{\beta}_{3}^{\prime} \vec{X}_{3}=\frac{3}{5} X_{1}+\frac{1}{5} X_{2}+\frac{1}{5} X_{3}$. One 
could achieve the objective simply by examining the majorization orders. In this example, one could easily find that $\vec{\beta}_{3}$ majorizes $\vec{\alpha}_{3}$ (see Example 1). Thereafter, applying Theorem 7, we obtain $\vec{\alpha}_{3}^{\prime} \vec{X}_{3} \succeq_{2} \vec{\beta}_{3}^{\prime} \vec{X}_{3}$.

If one does not want to apply Theorem 7 directly, one could consider applying Theorem 3 and Theorem 5 instead. We illustrate the procedure as follows: By Theorem 5, we know that since $\left(\frac{3}{5} \frac{1}{5} 0\right)$ majorizes $\left(\frac{2}{5} \frac{2}{5} 0\right)^{11}$, we have $\frac{2}{5} X_{1}+\frac{2}{5} X_{2} \succeq_{2} \frac{3}{5} X_{1}+\frac{1}{5} X_{2}$. By Theorem 3, adding another independent random variable, say $\frac{1}{5} X_{3}$, on both sides of the above does not alter the stochastic dominance relationship. Thus, we have $\frac{2}{5} X_{1}+\frac{2}{5} X_{2}+\frac{1}{5} X_{3} \succeq_{2}$ $\frac{3}{5} X_{1}+\frac{1}{5} X_{2}+\frac{1}{5} X_{3}$

Can the iid assumption be dropped in the diversification problem and the completely diversified portfolio be still optimal? Samuelson (1967) tells us that the answer is NO in general. He further establishes some results to relax the iid assumption. In this paper, we complement Samuelson's work by extending the results stated in the above theorems and corollaries by relaxing the independent and identically distributed condition as stated in the following corollaries:

Corollary 9 For $n>1$, let $\vec{X}_{n}=\left(X_{1}, \cdots, X_{n}\right)^{\prime}$ be a series of random variables that could be dependent. For any $\vec{\alpha}_{n}$ and $\vec{\beta}_{n}$,

$$
\vec{\alpha}_{n}^{\prime} \vec{X}_{n} \succeq_{2} \vec{\beta}_{n}^{\prime} \vec{X}_{n}
$$

if there exist $\vec{Y}_{n}$ and $A_{n n}$ such that $\vec{Y}_{n}=\left(Y_{1}, \cdots, Y_{n}\right)^{\prime}$ in which $\left\{Y_{1}, \cdots, Y_{n}\right\}$ are independent and identically distributed, $\vec{X}_{n}=A_{n n} \vec{Y}_{n}$, and

$$
\vec{\beta}_{n}^{\prime} A_{n n} \succeq_{M} \vec{\alpha}_{n}^{\prime} A_{n n}
$$

\footnotetext{
${ }^{11}$ Readers may refer to footnote 9 that it is not necessary that the summation of all weights in the vectors be 1 . As long as the summations of the weights are equal for all portfolios being compared, the results for Theorem 7 hold.
} 
where $\vec{\alpha}_{n}^{\prime} A_{n n}, \vec{\beta}_{n}^{\prime} A_{n n} \in S_{n}$.

Corollary 10 For $n>1$, let $\vec{X}_{n}=\left(X_{1}, \cdots, X_{n}\right)^{\prime}$ and $\vec{Y}_{n}=\left(Y_{1}, \cdots, Y_{n}\right)^{\prime}$ be two series of random variables which could be dependent. For any $\vec{\alpha}_{n}$ and $\vec{\beta}_{n}$,

$$
\vec{\alpha}_{n}^{\prime} \vec{X}_{n} \succeq_{2} \vec{\beta}_{n}^{\prime} \vec{Y}_{n}
$$

if there exist $\vec{U}_{n}=\left(U_{1}, \cdots, U_{n}\right)^{\prime}, \vec{V}_{n}=\left(V_{1}, \cdots, V_{n}\right)^{\prime}, A_{n n}$, and $B_{n n}$ in which $\left\{U_{1}, \cdots, U_{n}\right\}$ and $\left\{V_{1}, \cdots, V_{n}\right\}$ are two series of independent and identically distributed random variables such that $\vec{X}_{n}=A_{n n} \vec{U}_{n}, \vec{Y}_{n}=B_{n n} \vec{V}_{n}, U_{i} \succeq_{2} V_{i}$ for all $i=1,2, \ldots, n$; and

$$
\vec{\beta}_{n}^{\prime} B_{n n} \succeq_{M} \vec{\alpha}_{n}^{\prime} A_{n n}
$$

where $\vec{\alpha}_{n}^{\prime} A_{n n}, \vec{\beta}_{n}^{\prime} B_{n n} \in S_{n}$.

One could simply apply Theorem 7 to obtain the results of Corollary 9 and apply Theorem 3 to obtain the results of Corollary 10. One could then apply Theorem 1 to the above corollaries to obtain the following results:

Corollary 11 For $n>1$, let $\vec{X}_{n}=\left(X_{1}, \cdots, X_{n}\right)^{\prime}$ be a series of random variables which could be dependent. For any $\vec{\alpha}_{n}$ and $\vec{\beta}_{n}$,

$$
\vec{\alpha}_{n}^{\prime} \vec{X}_{n} \succeq_{2} \vec{\beta}_{n}^{\prime} \vec{X}_{n}
$$

if there exist $\vec{Y}_{n}$ and $A_{n n}$ such that $\vec{Y}_{n}=\left(Y_{1}, \cdots, Y_{n}\right)^{\prime}$ in which $\left\{Y_{1}, \cdots, Y_{n}\right\}$ are independent and identically distributed, $\vec{X}_{n}=A_{n n} \vec{Y}_{n}$, and

$$
\vec{\beta}_{n}^{\prime} A_{n n} \stackrel{D}{\rightarrow} \vec{\alpha}_{n}^{\prime} A_{n n}
$$

where $\vec{\alpha}_{n}^{\prime} A_{n n}, \vec{\beta}_{n}^{\prime} A_{n n} \in S_{n}$. 
Corollary 12 For $n>1$, let $\vec{X}_{n}=\left(X_{1}, \cdots, X_{n}\right)^{\prime}$ and $\vec{Y}_{n}=\left(Y_{1}, \cdots, Y_{n}\right)^{\prime}$ be two series of random variables which could be dependent. For any $\vec{\alpha}_{n}$ and $\vec{\beta}_{n}$,

$$
\vec{\alpha}_{n}^{\prime} \vec{X}_{n} \succeq_{2} \vec{\beta}_{n}^{\prime} \vec{Y}_{n}
$$

if there exist $\vec{U}_{n}=\left(U_{1}, \cdots, U_{n}\right)^{\prime}, \vec{V}_{n}=\left(V_{1}, \cdots, V_{n}\right)^{\prime}, A_{n n}$, and $B_{n n}$ in which $\left\{U_{1}, \cdots, U_{n}\right\}$ and $\left\{V_{1}, \cdots, V_{n}\right\}$ are two series of independent and identically distributed random variables such that $\vec{X}_{n}=A_{n n} \vec{U}_{n}, \vec{Y}_{n}=B_{n n} \vec{V}_{n}, U_{i} \succeq_{2} V_{i}$ for all $i=1,2, \ldots, n$; and

$$
\vec{\beta}_{n}^{\prime} B_{n n} \stackrel{D}{\rightarrow} \vec{\alpha}_{n}^{\prime} A_{n n}
$$

where $\vec{\alpha}_{n}^{\prime} A_{n n}, \vec{\beta}_{n}^{\prime} B_{n n} \in S_{n}$.

We provide the following examples to illustration the usefulness of the above corollaries.

Example 6 Let $Y_{1}, Y_{2}$ and $Y_{3}$ be independent and identically distributed random variables. Consider $X_{1}=\frac{3}{5} Y_{1}+\frac{1}{5} Y_{2}+\frac{1}{5} Y_{3}, X_{2}=\frac{1}{3} Y_{1}+\frac{1}{3} Y_{2}+\frac{1}{3} Y_{3}$, and $X_{3}=\frac{3}{4} Y_{1}+\frac{1}{8} Y_{2}+\frac{1}{8} Y_{3}$. Obviously, $\left\{X_{i}\right\}(i=1,2,3)$ are dependent. Corollary 9 assures that $\vec{\alpha}_{3}^{\prime} \vec{X}_{3} \succeq_{2} \vec{\beta}_{3}^{\prime} \vec{X}_{3}$ where $\vec{\alpha}_{3}=\left(\frac{4}{5}, \frac{1}{10}, \frac{1}{10}\right)^{\prime}, \vec{\beta}_{3}=\left(\frac{5}{6}, \frac{1}{12}, \frac{1}{12}\right)^{\prime}$, and $\vec{X}_{3}=\left(X_{1}, X_{2}, X_{3}\right)^{\prime}$. It is because one could easily find

$$
A_{33}=\left[\begin{array}{ccc}
\frac{3}{5} & \frac{1}{5} & \frac{1}{5} \\
\frac{1}{3} & \frac{1}{3} & \frac{1}{3} \\
\frac{3}{4} & \frac{1}{8} & \frac{1}{8}
\end{array}\right]
$$

such that

$$
\vec{\beta}_{3}^{\prime} A_{33} \succeq_{M} \vec{\alpha}_{3}^{\prime} A_{33}
$$

and $\vec{\alpha}_{3}^{\prime} A_{33}, \vec{\beta}_{3}^{\prime} A_{33} \in S_{3}$.

In addition, one could also apply Corollary 11 to assure $\vec{\alpha}_{3}^{\prime} \vec{X}_{3} \succeq_{2} \vec{\beta}_{3}^{\prime} \vec{X}_{3}$ as one could easily observe that

$$
\vec{\beta}_{n}^{\prime} A_{n n} \stackrel{D}{\rightarrow} \vec{\alpha}_{n}^{\prime} A_{n n}
$$


Example 7 Let $\left\{V_{i}\right\}(i=1,2,3)$ be a series of independent and identically distributed random variables with mean $\mu_{V}$ and variance $\sigma_{V}^{2}$, respectively. Let $U_{i}=p+q V_{i}(i=1,2,3)$ such that $0 \leq q<1$ and $p /(1-q) \geq \mu_{V}$. Then, by Theorem 8 in Li and Wong (1999), we have $U_{i} \succeq_{2} V_{i}(i=1,2,3)$.

Let $X_{1}=\frac{1}{2} U_{1}+\frac{1}{4} U_{2}+\frac{1}{4} U_{3}, X_{2}=\frac{1}{4} U_{1}+\frac{1}{3} U_{2}+\frac{5}{12} U_{3}, X_{3}=\frac{1}{6} U_{1}+\frac{1}{2} U_{2}+\frac{1}{3} U_{3}$, $Y_{1}=\frac{1}{3} V_{1}+\frac{1}{3} V_{2}+\frac{1}{3} V_{3}, Y_{2}=\frac{4}{7} V_{1}+\frac{1}{4} V_{2}+\frac{5}{28} V_{3}$, and $Y_{3}=\frac{4}{5} V_{1}+\frac{4}{25} V_{2}+\frac{1}{25} V_{3}$. Obviously, $\left\{X_{i}\right\}$ and $\left\{Y_{i}\right\}(i=1,2,3)$ are dependent.

Corollary 10 assures that $\vec{\alpha}_{3}^{\prime} \vec{X}_{3} \succeq_{2} \vec{\beta}_{3}^{\prime} \vec{Y}_{3}$ where $\alpha_{3}=\left(\frac{2}{3}, \frac{1}{6}, \frac{1}{6}\right)^{\prime}, \beta_{3}=\left(\frac{3}{4}, \frac{1}{8}, \frac{1}{8}\right), \vec{X}_{3}=$ $\left(X_{1}, X_{2}, X_{3}\right)^{\prime}$, and $\vec{Y}_{3}=\left(Y_{1}, Y_{2}, Y_{3}\right)^{\prime}$. It is because one could easily find

$$
A_{33}=\left[\begin{array}{ccc}
\frac{1}{2} & \frac{1}{4} & \frac{1}{4} \\
\frac{1}{4} & \frac{1}{3} & \frac{5}{12} \\
\frac{1}{6} & \frac{1}{2} & \frac{1}{3}
\end{array}\right] \quad \text { and } \quad B_{33}=\left[\begin{array}{ccc}
\frac{1}{3} & \frac{1}{3} & \frac{1}{3} \\
\frac{4}{7} & \frac{1}{4} & \frac{5}{28} \\
\frac{4}{5} & \frac{4}{25} & \frac{1}{25}
\end{array}\right]
$$

such that

$$
\beta_{3}^{\prime} B_{33} \succeq_{M} \alpha_{3}^{\prime} A_{33}
$$

and $\vec{\alpha}_{3}^{\prime} A_{33}, \vec{\beta}_{3}^{\prime} B_{33} \in S_{3}$

In addition, one could also apply Corollary 12 to assure $\vec{\alpha}_{3}^{\prime} \vec{X}_{3} \succeq_{2} \vec{\beta}_{3}^{\prime} \vec{Y}_{3}$ as one could easily find that

$$
\vec{\beta}_{3}^{\prime} B_{33} \stackrel{D}{\rightarrow} \vec{\alpha}_{3}^{\prime} A_{33}
$$

As a consequence of the above corollaries, an additional methodology is provided by which investors can make comparisons among a wide range of different convex combinations of dependent assets in a multivariate setting to check their SSD preferences. The results allow investors to rank different convex combinations of dependent assets not only by second-order stochastic dominance but also by their corresponding vectors of allocations. Our results also permit investors to eliminate non-efficient portfolios so that our 
results could help investors in determining the second-order stochastic dominance efficient set.

\section{Concluding Remarks}

By incorporating the majorization theory, this paper presents several new results of interest on stochastic dominance. Specifically, we establish some basic relationships in the portfolio choice problem by using both majorization theory and stochastic dominance. We also provide the foundation for applying majorization theory and stochastic dominance to investors' choices under uncertainty. The results are general, but presumably they are applicable to investment decision theory and comparisons of diversification of assets in a multivariate setting. We give new conditions for stochastic comparisons among different portfolio choices and new necessary and sufficient conditions that characterize diversified portfolios to give higher expected utilities. Thus, risk-averse and non-satiable investors will increase their expected utilities as the diversification of the portfolio increases. Our findings bring together, under a common framework, a number of fairly general results about diversification that permit comparisons among them. Our results could also be used to demonstrate the optimality of diversification and to obtain the preference orderings of portfolios for different asset allocations. In addition, our findings also impose further restrictions on admissible portfolios on the efficient frontier, and thus, our findings could also be used in determining the second-order stochastic dominance efficient set.

Nonetheless, Rotschild and Stiglitz (1970) show that for any two distributions with same mean, mean preserving spread and SSD are equivalent whereas Shalit and Yitzhaki (1994) verify that under some conditions, marginal conditional stochastic dominance is equivalent to SSD. Thus, incorporating the theory developed in our paper, one could conclude that under some regularity conditions, the preferences obtained from Dalton Transfer, majorization, and stochastic dominance could be equivalent to those obtained from mean preserving spread and marginal conditional stochastic dominance. 
Unlike the SD approach that is consistent with utility maximization, the dominance findings using the mean-variance criterion $^{12}$ may not be consistent with utility maximization if the assets returns are not normally distributed or investors' utilities are not quadratic. However, under some specific conditions, the mean-variance optimality could be consistent with the SD approach with utility maximization. For example, Meyer (1987), Wong $(2006,2007)$ and Wong and Ma (2008) have shown that if the returns of assets follow the same location-scale family, then a mean-variance domination could infer preferences by risk averters on the dominant fund to the dominated one. In addition, the Markowitz mean-variance optimization is equivalent to minimizing variance subject to obtaining a predetermined level of expected gain, see, for example, Bai, et al (2009). Thus, by incorporating the results developed in this paper and under some regularity conditions, the efficient set derived from Dalton Transfer, majorization and stochastic dominance could consequence to the same efficient set obtained from the mean-variance criterion and risk minimization. Further research could study their relationships in detail.

Lastly, we note that, recently other studies have findings that extend the bivariate framework of the diversification of risky assets to a multivariate setting. For example, Ma (2000) has removed the assumption of independence and studied the possibility that the random variables are exchangeable. In addition, Pellerey and Semeraro (2005) derive some new results for the portfolio choice problem when risky opportunities are correlated. They give new conditions for stochastic comparison among different portfolio choices and new necessary and sufficient conditions that characterize the portfolio to give the maximal expected utility. One avenue for further research would be to incorporate the findings in our paper to extend the results developed by Ma (2000), Pellerey and Semeraro (2005), and others. Another avenue for further research would apply the theory developed in this paper to different areas in Business, Economics and Finance. For example, one could incorporate the theory in this paper to explain well-known financial phenomena or

\footnotetext{
${ }^{12}$ see, for example, Markowitz (1952), Joro and Na (2006), Buckley, et al (2008), Josa-Fombellida and Rincón-Zapatero (2008), Zhao and Ziemba (2008) for more information.
} 
financial anomalies ${ }^{13}$ and to model investment risk. ${ }^{14}$

\footnotetext{
${ }^{13}$ see, for example, Wong and Bian (2000), Post (2003), Post and Levy (2005), Fong, et al. (2005), Fong and Wong (2006), and Post (2008).

${ }^{14}$ see, for example, Matsumura, et al. (1990), Seppälä (1994), Wong and Chan (2004), Gasbarro, et al (2007), Wong, et al (2001, 2008), and Lozano and Gutiérrez (2008).
} 


\section{References}

Ash, R.B., (1972), Real Analysis and Probability, New York, Academic Press.

Bai, Z.D., Liu, H.X., Wong, W.K., (2009), Enhancement of the Applicability of Markowitz's Portfolio Optimization by Utilizing Random Matrix Theory, Mathematical Finance, (forthcoming).

Bawa, V.S., Bodurtha, J.N. Jr., Rao, M.R., Suri, H.L., (1985), On Determination of Stochastic Dominance Optimal Sets, Journal of Finance, 40(2), 417-431.

Broll, U., Wahl, J.E., Wong, W.K., (2006), Elasticity of Risk Aversion and International Trade, Economics Letters, 92(1), 126-130.

Buckley, I., Saunders, D., Seco, L., (2008), Portfolio optimization when asset returns have the Gaussian mixture distribution, European Journal of Operational Research, 185(3), 1434-1461.

Clark, E., Jokung, O., (1999), A Note on Asset Proportions, Stochastic Dominance, and the 50\% Rule, Management Science 45(12), 1724-1737.

Dekel, E., (1989), Asset Demands Without the Independence Axiom, Econometrica 57(1), 163-169.

Feldstein, M.S., (1969), Mean Variance Analysis in the Theory of Liquidity Preference and Portfolio Selection, Review of Economic Studies, 36, 5-12.

Fishburn, P.C., (1974), Convex Stochastic Dominance with Continuous Distribution Functions, Journal of Economic Theory 7, 143-158.

Fong, W.M., Wong, W.K., (2006), The Modified Mixture of Distributions Model: A Revisit, Annals of Finance 2(2), 167- 178.

Fong, W.M., Wong, W.K., Lean, H.H., (2005), Stochastic Dominance and the Rationality of the Momentum Effect Across Markets, Journal of Financial 
Markets, 8, 89-109.

Gasbarro, D., Wong, W.K, Zumwalt, J.K., (2007), Stochastic dominance analysis of ishares. European Journal of Finance, 13, 89-101.

Hadar J., Russell, W.R., (1969), Rules for Ordering Uncertain Prospects, American Economic Review 59, 25-34.

Hadar J., Russell, W.R., (1971), Stochastic Dominance and Diversification, Journal of Economic Theory 3, 288-305.

Hanoch, G., Levy, H., (1969), The Efficiency Analysis of Choices involving Risk, Review of Economic Studies 36, 335-346.

Hardy, G.H., Littlewood, J.E., Pólya, G., (1934), Inequalities, Cambridge University Press, Cambridge, MA.

Ingersoll, J.E., (1987), Theory of Financial Decision Making, Rowman \& Littlefield, Totowa.

Joro, T., Na, P., (2006), Portfolio performance evaluation in a mean-varianceskewness framework, European Journal of Operational Research 175(1), 446-461.

Josa-Fombellida, R., Rincón-Zapatero, J.P., (2008), Mean-variance portfolio and contribution selection in stochastic pension funding, European Journal of Operational Research 187(1), 120-137.

Leung, P.L., Wong, W.K., (2008), On testing the equality of the multiple Sharpe Ratios, with application on the evaluation of iShares, Journal of Risk, 10(3), 1-16.

Levy, H., (2006), Stochastic Dominance: Investment Decision Making Under Uncertainty. Springer Publishers.

Levy, H., Markovitz, H.M., (1979), Approximating Expected Utility by a Func- 
tion of Mean and Variance, American Economic Review, 69(3), 308-317.

Li, C.K., Wong, W.K., (1999), Extension of Stochastic Dominance Theory to Random Variables, RAIRO Recherche Opérationnelle 33, 509-524.

Lozano, S., Gutiérrez, E., (2008), Data envelopment analysis of mutual funds based on second-order stochastic dominance, European Journal of Operational Research, 189(1), 230-244.

Ma, C., (2000), Convex Orders for Linear Combinations of Random Variables, Journal of Statistical Planning and Inference 84, 11-5.

Machina, M.J., (1982), Expected Utility Analysis without the Independence Axiom, Econometrica 50(2), 277-323.

Markowitz, H.M., (1952), Portfolio Selection, Journal of Finance, 7, 77-91.

Marshall, A.W., Olkin, I., (1979), Inequalities: Theory of Majorization and its Applications. San Diego: Academic Press.

Matsumura, E.M., Tsui, K.W., Wong, W.K., (1990), An extended multinomialDirichlet model for error bounds for dollar-unit sampling, Contemporary Accounting Research, 6, 485-500.

Meyer, J., (1987), Two-Moment Decision Models and Expected Utility Maximization, American Economic Review, 77(3), 421-30.

Ok, E., Kranich, L., (1998), The Measurement of Oppportunity Inequality: a Cardinality-Based Approach, Social Choice Welfare 15, 263-287.

Ortobelli Lozza, S., (2001), The Classification of Parametric Choices under Uncertainty: Analysis of the Portfolio Choice Problem, Theory and Decision 51, 297-327.

Pellerey, F., Semeraro, P., (2005), A Note on the Portfolio Selection Problem, Theory and Decision 59, 295-306. 
Post, T., (2003), Empirical Tests for Stochastic Dominance Efficiency, Journal of Finance, 58, 1905-1931.

Post, T., (2008), On the Dual Test for SSD Efficiency: With an Application to Momentum Investment Strategies, European Journal of Operational Research 185(3), 1564-1573.

Post, T., Levy, H., (2005), Does Risk Loving Drive Asset Prices? Review of Financial Studies, 18(3), 925-953.

Roberts, A.W., Varberg, D.E., (1973), Convex Functions, New York: Academic Press.

Rothschild, M., Stiglitz, J.E., (1970), Increasing risk: I. A definition, Journal of Economic Theory 2, 225-243.

Samuelson, P.A., (1967), General Proof that Diversification Pays, Journal of Financial and Quantitative Analysis 2(1), 1-13.

Seppälä, J., (1994), The diversification of currency loans: A comparison between safety-first and mean-variance criteria, European Journal of Operational Research, 74(2), 325-343.

Shalit, H., Yitzhaki, S., (1994), Marginal conditional stochastic dominance, Management Science 40(5), 670-684.

Tesfatsion, L., (1976), Stochastic Dominance and Maximization of Expected Utility; Review of Economic Studies 43, 301-315.

Tobin, J., (1958), Liquidity Preference and Behavior towards Risk, Review of Economic Studies, 25, 65-86.

Von-Neumann, J., Morgenstern, O., (1944), Theory of Games and Economic Behavior, Princeton University Press, Princeton N.J.

Wong, W.K., (2006), Stochastic Dominance Theory for Location-Scale Family, 
Journal of Applied Mathematics and Decision Sciences, 1-10.

Wong, W.K., (2007), Stochastic Dominance and Mean-Variance Measures of Profit and Loss for Business Planning and Investment, European Journal of Operational Research 182, 829-843.

Wong, W.K., Bian, G., (2000), Robust Bayesian inference in asset pricing estimation, Journal of Applied Mathematics \& Decision Sciences 4, 65-82.

Wong, W.K., Chan, R., (2004), The Estimation of the Cost of Capital and its Reliability, Quantitative Finance 4(3), 365 - 372.

Wong, W.K., Chan, R., (2008), Markowitz and Prospect Stochastic Dominances, Annals of Finance, 4(1), 105-129.

Wong, W.K., Chew B.K., Sikorski, D., (2001), Can P/E ratio and bond yield be used to beat stock markets? Multinational Finance Journal, 5(1), 59-86.

Wong, W.K., Li, C.K., (1999), A Note on Convex Stochastic Dominance Theory, Economics Letters 62, 293-300.

Wong, W.K., Ma, C., (2008), Preferences over Meyer's location-scale family, Economic Theory 37(1), 119-146.

Wong, W.K., Phoon, K.F., Lean, H.H., (2008), Stochastic dominance analysis of Asian hedge funds, Pacific-Basin Finance Journal, 16(3), 204-223.

Zhao, Y., Ziemba, W.T., (2008), Calculating risk neutral probabilities and optimal portfolio policies in a dynamic investment model with downside risk control, European Journal of Operational Research 185(3), 1525-1540. 